\title{
Cooperativas, fundaciones, sociedades civiles en Centroamérica. Posibilidades de transformación en entes más ágiles
}

\author{
Roxana Sánchez Boza \\ Catedrática de la Universidad de Costa Rica y de la Universidad Latina
}

Recibido: 01.07.2013

Aceptado: 30.07.2013

Sumario: I. Estado de situación de las leyes cooperativas y de organizaciones sin fines de lucro en Centroamérica. Normativa fundacional. II. El método comparativo para afrontar el sustento cooperativo. III. Transformación y constitución de entidades cooperativas bajo otros regímenes no cooperativos. Las sociedades civiles en los códigos civiles centroamericanos. IV. Conclusión. V. Bibliografía y otras fuentes.

Resumen: Se analizan las legislaciones cooperativas, de fundaciones y códigos civiles de la región centroamericana y República Dominicana. Se han promovido leyes que integran las ONG s, fundaciones y cooperativas. Únicamente es posible la comparación en cuanto a las posibilidades que tienen las fundaciones en Costa Rica, en cuanto realizar actividades lucrativas en aras a fortalecer su patrimonio.

Palabras clave: cooperativas, fundaciones, sociedades civiles, transformación, organizaciones sin fin de lucro.

Abstract: This paper examines the legislations of cooperatives and foundations and the civil codes of the Central American region and the Dominican Republic. A number of laws have been promoted that integrate NGOs, foundations and cooperatives. Comparison is only possible in terms of the possibilities that foundations in Costa Rica have regarding lucrative activities to strengthen their heritage.

Key words: cooperatives, foundations, civil societies, transformation, non-profit making organisations. 


\section{Estado de situación de las leyes cooperativas y de organizaciones sin fines de lucro en Centroamérica. Normativa fundacional}

En Centroamérica no se ha dado el fenómeno jurídico de una transformación de una cooperativa en fundación ${ }^{1}$. Existe la posibilidad de buscar opciones de formas organizativas más ágiles, que no persigan fines de lucro, como ha sucedido en Nicaragua con la Ley general sobre las personas jurídicas sin fines de lucro $^{2}$, en los dos primeros artículos de la ley se definen este tipo de personas:

«Artículo 1.-El objeto de la presente Ley es regular la constitución, autorización, funcionamiento y extinción de las Personas Jurídicas civiles y religiosas que sin fines de lucro existan en el país y de las que en el futuro se organicen.

Artículo 2.-Las Asociaciones, Fundaciones, Federaciones y Confederaciones, sin fines de lucro, sean civiles o religiosas, gozarán de personalidad jurídica una vez que llenen los requisitos establecidos en esta Ley.»

O bien dentro del conocido Sector de economía social muy desarrollado en Costa Rica ${ }^{3}$, donde han surgido las asociaciones solidaristas como un híbrido entre las cooperativas ${ }^{4}$ y asociaciones civiles ${ }^{5}$.

En el caso de Panamá se ha puesto la atención en las fundaciones como uno de los organismos auxiliares del cooperativismo reconocido expresamente por ley, dirigido a la ejecución de diversos objetivos beneficiosos para el movimiento cooperativo panameño, el artículo 24 de la Ley de cooperativas indica:

"Se consideran entidades auxiliares del cooperativismo, las asociaciones, fundaciones, sociedades y cualquier otra persona jurídica sin fines de lucro, nacionales o internacionales, debidamente recono-

1 Tampoco fue contemplado un caso tan particular como es la transformación de una fundación en una cooperativa en la Ley marco para las cooperativas de América Latina, Ed. Por la Alianza Cooperativa Internacional, texto elaborado por grupo de expertos Dante Cracogna, Roxana Sánchez Boza, Belisario Guarín, colaborador Henry Hagen San José, Costa Rica, 2009.

2 Ley general sobre las personas jurídicas sin fines de lucro, 1992, Managua Nicaragua.

3 Sánchez B. Roxana, El proyecto de creación del sector de economía laboral, tesis presentada en el Programa Centroamericano de Sociología, Universidad de Costa Rica, 1986, Costa Rica.

4 Ley de asociaciones solidaristas número 6790, publicada en «LA GACETA» N. ${ }^{\circ} 227$ del 28 de noviembre de 1984, San José Costa Rica.

5 Ley de asociaciones número 138 del 8 de agosto de 1939, San José, Costa Rica. 
cidas por IPACOOP, cuyos objetivos sean el fomento, financiamiento, educación, capacitación, asistencia técnica y, en general, el desarrollo del movimiento cooperativo».

En cada país de Centroamérica y El Caribe se encuentra una ley de cooperativas y de fundaciones, también se encuentra legislación específica sobre organizaciones sin fines de lucro, a las cuales se acercan las cooperativas como es el caso de Costa Rica que están exoneradas del pago del impuesto de renta, según artículo 63 de Ley de asociaciones cooperativas $^{6}$, en Honduras también hay una norma expresa sobre este tipo de exoneración, tal y como lo dispone la siguiente norma:

«Sección Sexta

Régimen tributario-prohibiciones y sanciones

Artículo 56.-Las cooperativas gozarán de las exenciones fiscales siguientes:

a) Exoneración de los impuestos fiscales que graven la renta, los bienes y las operaciones de las cooperativas. Se exceptúa el pago de las contribuciones para régimen de seguridad social; $\ldots\rangle^{7}$

En Honduras se permite transformación en otra cooperativa según el Título III, que trata de la integración cooperativa y considera la transformación como una manifestación de la misma, en sus artículos 82 a 87, a la par de otras modificaciones como son la fusión y la incorporación.

Es importante de anotar que únicamente dentro de la modalidad de las cooperativas es posible la transformación, por lo cual la ley hondureña prohíbe el cambio de un tipo de persona jurídica a otra, como sería que la cooperativa se convirtiera en fundación y viceversa. Al respecto es importante conocer textualmente el artículo siguiente:

«Artículo 85.—Una cooperativa sólo podrá transformarse en otra cooperativa.»

En El Salvador, República Dominicana ${ }^{8}$ al igual que los otros países de Centroamérica y El Caribe se limita el uso de la denominación «cooperativa» únicamente a las organizaciones de ese tipo y se obliga a

${ }^{6}$ Ley de Asociaciones Cooperativas N. ${ }^{\circ} 4179$ del 22 de agosto de 1968 y sus reformas, San José, Costa Rica.

7 Ley de cooperativas: Decreto Legislativo 65/87 (del 30 de abril de 1987). Honduras.

8 Ver Ley de cooperativas del 27 de enero de 1964. 
aclarar el nombre según el nivel de integración que le corresponda a la cooperativa -artículo 13- y se limita el nombre en razón de una protección de terceros relacionados con las cooperativas de ese país, tal y como lo vemos en el siguiente artículo 14:

«Las cooperativas no adoptarán denominaciones que induzcan a suponer un campo de operaciones distinto del previsto en los Estatutos, ni la existencia de un propósito contrario a las prohibiciones que establece la Ley.»

En Costa Rica existe la Ley de asociaciones cooperativas y creación del instituto nacional de fomento cooperativo, que data del 30 de abril de 1982.

En Costa Rica se profundiza mucho en el aspecto asociativo de la cooperativa, tanto así que se le denomina "asociación cooperativa» y por el artículo primero de la ley indicada se declara de conveniencia y utilidad pública y de interés social, la constitución y funcionamiento de asociaciones cooperativas, por ser uno de los medios más eficaces para el desarrollo económico, social, cultural y democrático de los habitantes del país.

Además no se permite el uso de la palabra cooperativa si la entidad no lo es, el artículo 7 reza:

«A ninguna entidad, firma, corporación o asociación que no se ajuste rigurosamente a las formalidades prescritas en esta Ley, cualesquiera que sean sus actividades, les será permitido usar la bandera o el emblema internacional o nacional de la cooperación, ni adoptar la denominación "Cooperativa» u otra análoga que pudiera inducir a error, ni insertarlas en su razón social o en sus títulos ni usarlos en forma alguna en sus documentos, papelería, avisos o publicaciones. Durante el período de organización de una cooperativa deberá ésta adoptar dicha denominación pero agregando la frase "en formación». Para estos efectos el Instituto Nacional de fomento Cooperativo que esta ley crea, llevará un registro de las cooperativas en formación y señalará en cada caso el lapso durante el cual podrán hacer uso de la facultad que otorga este artículo.»

De una búsqueda a profundidad se encuentra un sólo ejemplo de transformación de cooperativas, para el caso específico de cooperativas de producción de bienes: el artículo 130 explica esa posibilidad.

«Los trabajadores de cualquier cooperativa de producción de bienes podrán solicitar su ingreso como asociados de conformidad con 
lo establecido en el artículo 58 de esta Ley. Cuando por lo menos un $60 \%$ del total de trabajadores de una cooperativa, asociados o no, solicite al INFOCOOP la transformación de una cooperativa de cogestión, el INFOCOOP deberá efectuar los estudios correspondientes para determinar la viabilidad, posibilidad y utilidad de la transformación. En caso de que los resultados del estudio sean favorables a juicio del INFOCOOP, será obligatorio para la cooperativa su transformación en cooperativa de cogestión.»

En Honduras solo quienes sigan la Ley de cooperativas pueden utilizar la palabra cooperativa en su nombre.

"Artículo 8.- Solamente las asociaciones que se constituyan de conformidad a esta ley, tendrán derecho de incluir las expresiones "Asociación Cooperativa", u otras semejantes en su denominación o razón social, nombre comercial, documentos, textos de propaganda o publicidad. Se exceptúan los casos en que tales términos sean utilizados como consecuencia de convenios intergubernamentales. Durante el período de organización de una asociación cooperativa podrá ésta adoptar dicha denominación, pero agregando las palabras "en formación"; y si fuere disuelta deberá conservarlo agregando la frase "en liquidación". Los infractores a lo dispuesto en los incisos anteriores, serán sancionados con multas de cien a quinientos colones, que impondrá gubernativamente la oficina que ejerza la vigilancia del Estado, previa audiencia por setenta y dos horas al sancionado. La Resolución que imponga la multa, admitirá apelación para ante el Ministerio de Economía, quien antes de resolver la alzada mandará oír por setenta y dos horas al apelante y a la autoridad sancionante. Lo dicho es sin perjuicio de las demás responsabilidades, de cualquier índole que éstas sean, a que hubiera lugar.»

Guatemala por su parte tiene una normativa no prohibitiva en su redacción y acoge en la ley general de cooperativas, decreto 82/78 del 7 de diciembre de 1978, en el artículo 6, la posibilidad del desarrollo de actividades requeridas para su crecimiento y comunes a sus miembros.

En la ley de cooperativas panameña no se encuentra ninguna referencia a la transformación, aunque tiene su capítulo de integración cooperativa -artículos 16 a 18 ibidem-.

No existen normas de interpretación que remitan a otro texto legal donde pueda aplicar la analogía en el caso de transformación de cooperativas en otras cooperativas $u$ otras personas jurídicas similares, como sería el hecho de no perseguir el lucro como objeto social preponderante. 
En Panamá en artículo 10 es obligatorio incluir la palabra cooperativa como parte del nombre social de la misma. Y en forma expresa el artículo 21 de la ley de cooperativas, permite la transformación interna, o sea de un tipo de cooperativa a otro, según la Ley del 1 mayo de 1997 Ley especial de cooperativas.

De importancia es la consideración de entes auxiliares del cooperativismo en cuanto a las fundaciones y otras asociaciones sin fines de lucro, siempre y cuando cumplan con los objetivos de fomento, financiamiento, educación, capacitación, asistencia técnica y en general el desarrollo del movimiento cooperativo, artículo 24 de la citada ley 17 de 1997:

«Se consideran entidades auxiliares del cooperativismo, las asociaciones, fundaciones, sociedades y cualquier otra persona jurídica sin fines de lucro, nacionales o internacionales, debidamente reconocidas por IPACOOP, cuyos objetivos sean el fomento, financiamiento, educación, capacitación, asistencia técnica y, en general, el desarrollo del movimiento cooperativo».

Cabe resaltar que la ley cooperativa panameña dejó prevista la utilización de la integración e interpretación jurídicas cuando existan lagunas en el ordenamiento jurídico en cooperativas en Panamá, como es el artículo 139 que indica que los casos no previstos por la ley cooperativa y su reglamento se resolverán de conformidad con el Derecho cooperativo, con el estatuto respectivo, la doctrina, los principios del cooperativismo, y en su defecto de acuerdo con las regulaciones del Derecho común que por su naturaleza y similitud puedan aplicarse a las cooperativas.

El artículo 39 de la Constitución Política de Panamá contempla la creación de organizaciones sin fines de lucro como las fundaciones, que no sean contrarias a la moral y al orden legal.

Es el Código Civil panameño el cuerpo normativo que desarrolla ese artículo y en el correspondiente al número 64 incisos b, contempla a las fundaciones de interés público, creadas y reconocidas por ley especial.

Podemos considerar algunos aspectos propios de las fundaciones en Panamá.

MORGAN Y MORGAN nos refiere que la iniciativa de crear la ley sobre fundaciones de interés privado en Panamá surge con motivo de la popularidad adquirida en Europa, especialmente en Suiza, por las fundaciones familiares de Liechtenstein.

En Panamá por Decreto Ejecutivo No. 417, de 8 de agosto de 1995, se crea en la Dirección General del Registro Público, la Sección de Fun- 
daciones de Interés Privado y se reglamenta la inscripción de la constitución, modificación y extinción de dichas Fundaciones. Lo anterior por mandato de la Ley de fundaciones de interés privado, que en el artículo 33 de la Ley. No. 25 de 12 de junio de 1995, establece que deberán practicarse en el Registro las inscripciones relacionadas con esta nueva institución jurídica.

Sin embargo, en relación con las fundaciones nacidas en el seno del Movimiento cooperativo, consideradas por mandato legal como organismos auxiliares del cooperativismo panameño, tales entes no se registran en su ciclo de vida y posible extinción en el mencionado registro, porque estamos frente a una legislación especial encontramos en diferentes normas. Así de manera expresa dispone el artículo 117 de la citada ley 17 de 1997, las cooperativas, las federaciones, la confederación, los organismos auxiliares y demás organismos cooperativos de que trata la misma, están sujetos a la fiscalización estatal, que será la encargada de velar porque los actos atinentes a su constitución, funcionamiento, cumplimiento de sus objetivos sociales, disolución y liquidación se ajusten a las normas legales y estatutarias.

Por su parte, el artículo 118 de la referida excepción legal es claro al señalar que la autoridad encargada de la aplicación de la legislación cooperativa y el órgano para la fiscalización pública de que trata el citado artículo 117 es el Instituto Panameño Autónomo Cooperativo.

Cabe agregar que el decreto ejecutivo 137 de 5 de noviembre de 2001, reglamentario de la ley 17 de 1997, desarrolla en su capítulo X la figura de las entidades auxiliares del cooperativismo, puntualizándose en el artículo 52 que las mismas complementan con actividades específicas a las organizaciones cooperativas y sus asociados en el desarrollo cooperativista del país, canalizando recursos técnicos, filosóficos y financieros. Por ello, conforme se establece en el artículo 54 del citado reglamento, el reconocimiento, inscripción y fiscalización de estas entidades es competencia del Instituto Panameño Autónomo Cooperativo (IPACOOP).

\section{a) Fundaciones de Cooperativas en Panamá}

En Panamá fue creada la Fundación para la vivienda cooperativa FUNDAVICO que tiene como principal objetivo dotar de vivienda al movimiento cooperativo de ese país. Corresponde al IPACOOP — Instituto panameño de cooperativas - su supervisión, en el tanto forma parte 
de la estructura del cooperativismo panameño, como ente auxiliar de cooperativas reconocido por ley especial ${ }^{9}$.

FUNDAVICO es una fundación inscrita en la Dirección de Registro de Cooperativas del Instituto Panameño Cooperativo, ente que le otorgó personería jurídica como ente auxilia del cooperativismo, mediante la resolución IPACOOP-PJ-11 de 4 de diciembre de 1989 según el artículo 24 de la citada ley 17 de 1997:

Otra fundación que funciona en el movimiento cooperativo panameño es la Fundación Centro Recreativo y Educativo - FUCER - creada por ley número 25 de 12 de junio de 1995, la cual fue constituida por la Cooperativa de ahorro y crédito El educador R.L. Coopeduc R.L

«Sus objetivos según el artículo tercero del acta fundacional son los siguientes:

a) Brindar facilidades educativas modernas de última tecnología para la realización de programas educativos, cursos, congresos, pasantías, proyectos, tutorías, consultorías, asambleas y otros eventos en diversas magnitudes, al sector cooperativo de manera particular y a la comunidad nacional e internacional en general.

b) Ofrecer al sector cooperativo y a la comunidad nacional e internacional, un complejo institucional que faciliten la integración y la convivencia humana en todos los ámbitos.

c) Crear otros servicios colaterales que complementen las actividades principales del centro.»

\section{b) República Dominicana}

En República Dominicana no se encuentra en la Ley de asociaciones cooperativas ninguna información sobre organismos cooperativos auxiliares ni sobre fundaciones para cooperativas.

\section{c) El Salvador}

Si existe en El Salvador la ley de asociaciones y fundaciones sin fines de lucro, Decreto N. ${ }^{\circ} 894$, la cual fue promulgada por la Asamblea

9 Ver criterio de la Procuraduría de la República panameña, emitido el 5 de mayo de 2008, número C38-08 sobre la validez de la supervisión a FUNDAVICO por parte del IPACOOP, según fuente electrónica http://www.procuraduriaadmon.gob.pa:8000/Busquedas/documento_consult?mime_t=application/pdf\&query_doc=select imagen from procadm.t_texto_consulta where numero_consulta C-034-08 \&a=C-034-08. 
Legislativa considerando que según la Constitución de la República, es obligación del Estado garantizar a los habitantes de El Salvador el derecho de asociarse libremente; y por la operación de asociaciones y fundaciones sin fines de lucro, cuyo esfuerzo y brinda trabajo con resultados positivos en el mejoramiento de las condiciones de vida de muchas familias salvadoreñas.

Otro de los objetivos del nacimiento de la ley en mención se relaciona con el deber del Estado de velar por la transparencia que en el manejo de sus fondos en la actividad de estas entidades; ya que parte de ellos provienen del público, así como de sus donantes ${ }^{10}$.

\section{d) Las Fundaciones en Costa Rica: Fundaciones de interés privado}

La ley de Fundaciones existe en Costa Rica desde el año 1973, N. 5338, en el artículo primero se establecen los objetivos que puede tener una fundación:

«Artículo 1.-Reconócele personalidad jurídica propia a las fundaciones, como entes privados de utilidad pública, que se establezcan sin fines de lucro y con el objeto de realizar o ayudar a realizar, mediante el destino de un patrimonio, actividades educativas, benéficas, artísticas o literarias, científicas, y en general todas aquellas que signifiquen bienestar social.»

Y en el artículo 7 se recalca que las fundaciones no tienen finalidades comerciales. Sin embargo podrán realizar operaciones de esa índole para aumentar su patrimonio, pero los ingresos que obtengan deberán destinarlos exclusivamente a la realización de sus propios objetivos.

La existencia de ese permiso a las fundaciones para realizar actividades lucrativas cuyo producto ingresa al patrimonio de la fundación, es un punto adelante respecto de España, lo interesante consiste en que la fundación no cambia de naturaleza jurídica pese a realizar actividades que no se contemplan en el artículo primero de la ley antes transcrita, existe una norma expresa que permite a las cooperativas establecer alianzas con entes no lucrativos, por lo que se podría pensar que en el caso de este país si es posible que una cooperativa constituya una fundación, como es el caso de Panamá, que a continuación exponemos.

10 Se encuentra el acta fundacional protocolizada en Notaría pública según referencia Fundacion Centro Recreativo y Educativo, ver referencia en la dirección http://www. fucerpanama.com/pdf/estatuto_fucer.pdf. 


\section{El método comparativo para afrontar el sustento cooperativo}

Con el fin de ofrecer al lector un hilo conductor se ha tomado como referente metodológico el Derecho Comparado, definido por Fernando Torres como:

«El estudio y propuestas de comparaciones, recepciones, trasplantes, migraciones, exportaciones, importaciones, reorganizaciones, fusiones, escisiones, transformaciones, integraciones, de cualquier institución jurídica, el cual puede ser aplicado a cualquier disciplina jurídica, dentro de las cuales podemos citar el caso del derecho privado, público y mixto, en cuyo caso estaremos frente a supuestos de derecho privado comparado, público amparado y mixto comparado $^{11}$ ».

Es de esa forma como el derecho comparado permite abordar un determinado tema, instituto, norma, figura jurídica, con un enfoque metodológico propio para el establecimiento de comparaciones que permiten delimitar similitudes, diferencias, beneficios y contextos del objeto de estudio.

Por su parte la doctora Milushka Rojas establece que el Derecho Comparado:

«... tiene como objeto de estudio confrontar los ordenamientos e instituciones jurídicas que existen en el mundo, analizar las diferencias y semejanzas de su estructura y las causas de esas relaciones, con el fin de promover y asegurar el progreso del derecho nacional12》.

La doctora Rojas destaca a la vez una función instrumental del Derecho comparado, que vendría como medio para promover y asegurar el progreso del derecho nacional.

Nuestra exposición ha seguido básicamente las pautas de la micro comparación, porque se ha realizado una búsqueda de la normativa en las leyes cooperativas, que nos den una idea acerca de cuáles son los alcances y limitaciones de su funcionamiento, sus posibilidades de aprovechar otras instituciones jurídicas para un mejor desarrollo empresarial y social así como dónde hay diferencias o similitudes que nos permitan determinar si hay posibilidades de descubrir lo verdaderamente comparable.

11 TORRES, F. (2013). Método comparativo, un estudio de derecho comparado.

12 ROJAS, M. (2013). Importancia del Derecho Comparado en el siglo XXI. 
Se comparan elementos formales: dados por legislación especial de cooperativas, las leyes relacionadas con fundaciones y los códigos civiles...

Identificación de los elementos de comparación fundaciones y cooperativas, su legislación especial y puntos de encuentro

Se ubica espacialmente la investigación en Centroamérica y República Dominicana, donde se encuentran países que ofrecen elementos para establecer la comparación.

Finalmente se toman datos de una propuesta realizada al Cooperativismo costarricense para ofrecer más elementos en la búsqueda de posibilidades de desarrollar la empresa cooperativa con figuras jurídicas más simples en su estructura y ágiles en la toma y ejecución de decisiones.

\section{Transformación y constitución de entidades cooperativas bajo otros regímenes no cooperativos. Las sociedades civiles en los códigos civiles centroamericanos}

En los Códigos Civiles de los países de Centroamérica y República Dominicana, los títulos dedicados a la sociedad civil la contemplan como un ente generador de ganancias mientras que en Costa Rica se da la posibilidad de constituir sociedades civiles con fines no lucrativos, lo que le asimila a una cooperativa, este es un punto de partida para ofrecer alternativas de organización económico-social más ágiles en su nacimiento y su administración.

España en la Ley de cooperativas del País Vasco dejó la posibilidad de transformar fundaciones en cooperativas.

La reforma de la Ley de Fundaciones de 1994 ha permitido la participación de las fundaciones en sociedades cooperativas, del tema se ha ocupado Argudo Périz.

El autor refiere de una reforma del año 2002 a la Ley especial de fundaciones que favorece que estas personas jurídicas utilicen de forma dinámica su patrimonio, y lo conviertan en un patrimonio de explotación, adoptando medios y técnicas empresariales para incrementar sus recursos y poder cumplir sus fines. En las fundaciones viene favorecido por la ausencia de propietarios, lo que favorece el cumplimiento de los fines, que se convierten, de algún modo, en propietarios del patrimonio.

Apunta Argudo Périz que: "La realización de actividades empresariales, mercantiles e industriales, es la cuestión que más recelos doctrinales y cautelas legales ha provocado, por la aparente contra- 
dicción entre realización de actividades que pueden generar beneficios económicos y la ausencia de ánimo de lucro que caracteriza a la persona jurídica fundación, por la posibilidad de desnaturalizar sus fines».

Manzano Malaxechevarría completa la idea de la transformación de una fundación en cooperativa cuando nos refiere el origen de la norma permisiva de tal transformación, cuando explica la ubicación de la norma y la forma de su comportamiento, en sus propias palabras lo encontramos:

«En la Ley 4/1993, de Cooperativas de Euskadi, modificada por la Ley 1/2000, de 29 de junio, al regular las modificaciones estatutarias en esta clase de entidades, indica cuáles son los requisitos que habrá de reunir la modificación de los estatutos de la fundación para transformarlos en los de una Cooperativa con respeto a lo establecido en su Ley rectora, es decir, con un capital social mínimo de tres mil euros (art. 4 LCE), aportado en moneda de curso legal, salvo que la Asamblea General o los Estatutos acuerden otra cosa (art. 57.3 LCE), con tres socios como mínimo (art. 19.1 LCE) y con cumplimiento de las exigencias que la propia Ley impone a fin de inscribir la constitución en el Registro de Cooperativas, inscripción que tiene carácter constitutivo y sin la cual no hay cooperativa posible.»

Como indicamos en el anterior aparte, la ley de cooperativas de Costa Rica no establece expresamente la posibilidad de la transformación de fundaciones en cooperativas, sin embargo existen posibilidades de considerar que otros esquemas jurídicos bastante ágiles por la simplicidad con que funcionan pueden ofrecer la posibilidad de establecer cooperativas con menos personas y con la toma de decisiones y su ejecución en forma más efectiva. Como punto de partida se analizó la figura de la sociedad civil en Costa Rica y se estableció que en la normativa del Código Civil se da la posibilidad de hacer una sociedad civil sin fines de lucro, no requiere de órgano fiscalizador, de junta directiva sino tan solo de un administrador, con lo cual grupos pequeños de empresarios podrían formar una sociedad civil con fines cooperativos y superar el número y la cantidad de órganos sociales y su integración abundante que actualmente tienen las cooperativas en Costa Rica.

Se aclara que para constituir una cooperativa tradicional se requieren 20 socios y una de trabajo asociado - denominada cooperativa de autogestión- requiere de 12 fundadores, mientras que en la sociedad civil, se requiere únicamente de dos personas para constituirla. 
La propuesta fue presentada al Movimiento cooperativo costarricense con ocasión del XII Congreso Nacional Cooperativo, en el Foro Jurídico: 27 de octubre de 2010. EDICOOP13, dentro del eje temáticojurídico 4: Redacción de la sociedad civil cooperativa, la cual pasamos a transcribir:

«Propuesta de Dra. Roxana Sánchez Boza.

La idea es ofrecer alternativas de organización económica-social para grupos pequeños, que en su inicio necesitan una estructura organizativa menos pesada que la ofrecida por una cooperativa porque en la sociedad civil sólo se requiere un administrador, que es el representante legal y actúa bajo los mandatos de la asamblea de socios, que fácilmente se puede reunir si son pocos.

En la doctrina cooperativa mundial las formas de organización cooperativa son de diferente tipo, la $\mathrm{ACl}$ las reconoce y acepta su incorporación.

En Costa Rica la Ley de Asociaciones Cooperativas en algunos casos indica que son asociaciones en otros sólo cooperativas, pero el aspecto más importante es que el artículo 164 de la Constitución Política no se limita únicamente a las asociaciones cooperativas.

En la normativa del INFOCOOP se contempla que éste debe impulsar proyectos de economía social y permite el control de los recursos a través de la figura de participación en los órganos de dirección.

La propuesta está dirigida a grupos de empresarios cuyo giro económico no requiere de un número mayor a cuatro miembros y que tienen el deseo y la mejor disposición de trabajar en forma cooperativa. También será posible establecer plazos para una incorporación posterior al régimen de asociaciones cooperativas, o bien modificar la ley para su reconocimiento pleno.

En síntesis les presento un cuadro comparativo de la organización y funcionamiento de ambas entidades.

13 Documento presentado a consideración del XII Congreso Nacional Cooperativo, año 2010, denominado «Resumen de acuerdos de los foros de sectores con aspectos o efectos juridicos. Elaborado por Roxana Sánchez Boza». San José Costa Rica, 2010. 


\section{Comparación de requisitos de inscripción y organización de sociedades civiles cooperativas y asociaciones cooperativas}

\begin{tabular}{|c|c|c|}
\hline $\begin{array}{l}\text { Formas organización } \\
\text { socio-económica }\end{array}$ & Sociedad civil cooperativa & Asociación cooperativa \\
\hline Requisitos constitución & $\begin{array}{l}\text { En dirección personas jurí- } \\
\text { dicas. Registro nacional }\end{array}$ & $\begin{array}{l}\text { Infocoop y ministerio tra- } \\
\text { bajo }\end{array}$ \\
\hline Número miembros & $\begin{array}{l}2 \\
\text { Se propone en este caso } \\
\text { un mínimo de cuatro. }\end{array}$ & 12 o 20 asociados \\
\hline Tipo miembros & Personas físicas o jurídicas & $\begin{array}{l}\text { Cooperativa base: perso- } \\
\text { na física }\end{array}$ \\
\hline Objeto social & De economía social & $\begin{array}{l}\text { Según sector económico } \\
\text { o modelo cooperativo }\end{array}$ \\
\hline Forma jurídica & $\begin{array}{l}\text { Escritura pública. Código } \\
\text { civil }\end{array}$ & $\begin{array}{l}\text { Acta constitutiva revisa- } \\
\text { da por INFOCOOP Ley de } \\
\text { asociaciones cooperativas }\end{array}$ \\
\hline Tiempo inscripción & Máximo un mes & Mínimo seis meses \\
\hline Estructura organizativa & $\begin{array}{l}\text { Asamblea y un represen- } \\
\text { tante o administrador }\end{array}$ & $\begin{array}{l}\text { Asamblea, consejo admi- } \\
\text { nistración comité educa- } \\
\text { ción, comité vigilancia, } \\
\text { gerente }\end{array}$ \\
\hline
\end{tabular}

Origen: Elaborado por Dra. Roxana Sánchez Boza

Para Foro Jurídico del XII Congreso Nacional Cooperativo

EDICOOP 27 de octubre de 2010.

La propuesta no fue acogida sin embargo, considero que es una posibilidad más de ampliar las figuras jurídicas que pueden servir de sombrilla al cooperativismo para lograr empresas cooperativas más ágiles y eficientes.

\section{Conclusión}

La propuesta de analizar las legislaciones cooperativas, de fundaciones y códigos civiles de la región centroamericana y República Dominicana indudablemente nos dio luces sobre los diferentes rumbos que se han tomado en ese ámbito espacial.

Se han promovido leyes que integran las ONG s, fundaciones y cooperativas o bien, es posible rescatar figuras jurídicas nacidas desde 
el Código Napoleón, como es el caso de Costa Rica y remozarlas en beneficio del desarrollo de la empresa cooperativa.

Únicamente es posible la comparación en cuanto a las posibilidades que tienen las fundaciones en Costa Rica, en cuanto realizar actividades lucrativas en aras de fortalecer su patrimonio y el caso del país vasco, como un avance en el tema base de este ensayo.

En cuanto a las similitudes encontradas con las leyes cooperativas y los códigos civiles analizados, vemos que responden a un modelo de unificación ya superado en el desarrollo histórico del Derecho Comparado, que más bien encierra y limita la evolución del cooperativismo centroamericano y dominicano. No hay duda que es importante una revisión de conceptos y sus fundamentos para decidir si se mantiene la normativa prohibitiva en las alianzas y acuerdos de las cooperativas para que estén en posición de igualdad y equidad con otras organizaciones que no persiguen el lucro como objetivo esencial de su existencia.

\section{Bibliografía y otras fuentes}

ARGUDO PÉRIZ, J.L., La participación de fundaciones en cooperativas, Diploma de especialización en dirección de organizaciones de economía social, E. U. Estudios sociales Universidad de Zaragoza.

CONACOOP, "Resumen de acuerdos de los foros de sectores con aspectos o efectos juridicos. Elaborado por Roxana Sánchez Boza». San José Costa Rica, 2010.

GONZÁLEZ, J.M., http://www.unizar.es/does/asignaturas/joseramongonzalez3.pdf.

MANZANO MALAXECHEVARRÍA, J.R., http://www.forulege.com/dokumentuak/juanra_manzano_dec_2_2013.pdf.

ROJAS, M. (2013), Importancia del Derecho Comparado en el Siglo XXI.

TORRES, F. (2013), Método comparativo, un estudio de derecho comparado.

Carta urbana: http://www.fundasal.org.sv/documentos/cartas_urbanas/carta_ urbana_152.pdf.

Criterio de Procuraduría de Panamá N. C38-05 en http://www.procuraduriaadmon.gob.pa:8000/Busquedas/documento_consult?mime_t=application/ pdf\&query_doc=select/imagen from procadm.t_texto_consulta/where numero_consulta' $C-034-08 \& a=C-034-08$

Acta Fundacional de la Fundación Centro Recreativo y Educativo http://www. fucerpanama.com/pdf/estatuto_fucer.pdf

Codigos Civiles, Leyes Especiales

Código Civil de El Salvador Decreto Ley de 23 de agosto de 1859.

Decreto Ley N. 106 del 14 de setiembre de 1963 de Guatemala. 
Panamá. Código Civil y sus reformas entro a regir el primero de julio de 1967. España. Ley 50/2002, de 26 de diciembre, de Fundaciones (BOE 310, de 27 de diciembre).

Ley marco para las cooperativas de América Latina, Ed. Por la Alianza Cooperativa Internacional, texto elaborado por grupo de expertos Dante Cracogna, Roxana Sánchez Boza, Belisario Guarín, con la colaboración de Henry Hagen. San José, Costa Rica, 2009, ver en http://www.ilo.org/public/spanish/ support/lib/resource/subject/coop/ley_marco_al.pdf.

Nicaragua: http://www.infocoop.gob.ni/index.php.

Honduras: http://www.honduraslegal.com/legislacion/civil.htm.

Documento presentado a consideración del XII Congreso Nacional Cooperativo, año 2010, denominado «Resumen de acuerdos de los foros de sectores con aspectos o efectos jurídicos. Elaborado por Roxana Sánchez Boza». San José Costa Rica, 2010.

Criterio de la Procuraduría de la República panameña, emitido el 5 de mayo de 2008, número C38-08 sobre la validez de la supervisión a FUNDAVICO por parte del IPACOOP, según fuente electrónica http://Www. procuraduriaadmon.gob.pa:8000/Busquedas/documento_consult?mime_t=application/ pdf\&query_doc=select imagen Fromm procadm.t_texto_consulta where numero_consulta C-034-08 \&a=C-034-08. 